\title{
Dual magnetic mass of a hot quark-gluon plasma
}

\author{
M. Baker* \\ Laboratoire de Physique Théorique et Hautes Energies, Université de Paris XI, Bâtiment 211, F-91405 Orsay CEDEX, France
}

James S. Ball

University of Utah, Salt Lake City, Utah 84112

F. Zachariasen

Theory Division, CERN, 1211 Geneva 23, Switzerland

(Received 22 May 1992; revised manuscript received 17 September 1992)

\begin{abstract}
The dual magnetic mass of a hot quark-gluon plasma is computed in the lowest order of dual QCD, which predicts a well-defined (dual) gauge-invariant result for it. This is because, in dual QCD, electricity and magnetism are interchanged, so magnetic calculations in dual QCD are easy if the corresponding electric ones in ordinary QCD are easy, and vice versa. We obtain the (leading-order) numerical result $\widetilde{m}_{\text {mag }}=\left(\frac{7}{12}\right)^{1 / 2} g T$ for the dual magnetic mass, where $g$ is the dual (color-magnetic) coupling constant.
\end{abstract}

PACS number(s): 12.38.Mh, 12.38.Aw, 12.38.Bx

The "electric mass," the electric plasma frequency, and the electric Landau damping rate of a hot quark-gluon plasma were calculated long ago in the high-temperature limit $[1,2]$. To leading order the electric mass is [1]

$$
m_{\mathrm{el}}^{2}=e^{2} T^{2}\left(1+N_{f} / 6\right),
$$

for $N_{f}$ quark flavors, the plasma frequency is [1] $\omega_{\mathrm{el}}=m_{\mathrm{el}} / \sqrt{3}$, and the Landau damping is [2]

$$
\gamma_{\mathrm{el}}=-\frac{3 e^{2} T}{4 \pi} \ln e
$$

(we use $e$ for the quark-gluon coupling constant, so that $\alpha_{s}=e^{2} / 4 \pi$ ). All of these quantities are generally believed to be gauge invariant [3].

The order-eT contribution to $m_{\mathrm{el}}$ is gauge invariant and computable in perturbation theory. It gives the correlation length for the color-electric field [1]. Correspondingly, the magnetic mass of a quark-gluon plasma begins only in order $e^{2} T$, with a perturbatively uncalculable numerical coefficient. The magnetic mass remains an enigma in conventional QCD, and therefore nothing is known about the correlation function of the colormagnetic induction.

The purpose of this Brief Report is to point out that the situation described above is reversed in dual QCD, in which QCD is expressed in terms of the electric vector potentials $C_{\mu}^{a}$ rather than the conventional magnetic potential $A_{\mu}^{a}$ [4]. In this language the dual field tensor

$$
G_{\mu v}=\partial_{\mu} C_{v}-\partial_{v} C_{\mu}-i g\left[C_{\mu}, C_{v}\right],
$$

where $g$ is the magnetic coupling constant, related (in the

\footnotetext{
${ }^{*}$ On leave of absence from Univ. of Washington, Seattle, WA 98105 .
}

infrared limit) to the ordinary QCD coupling constant $e$ by the Dirac quantization condition $e g=2 \pi$. In terms of $G_{\mu \nu}$, the electric displacement is $D_{i}=\frac{1}{2} \epsilon_{i j k} G_{j k}$ and the magnetic field is $H_{i}=G_{0 i}$, in contrast to the usual field tensor

$$
F_{\mu \nu}=\partial_{\mu} A_{v}-\partial_{v} A_{\mu}-i e\left[A_{\mu}, A_{v}\right],
$$

in which the electric field is $E_{i}=F_{0 i}$ and the magnetic induction is $B_{i}=\frac{1}{2} \epsilon_{i j k} F_{j k}$.

In dual QCD, the poles of the dual gluon propagator yield the dual electric and magnetic masses $\widetilde{m}_{\mathrm{el}}$ and $\widetilde{m}_{\text {mag }}$. As $m_{\text {el }}$ and $m_{\text {mag }}$ in ordinary QCD can be associated with the correlation functions of the electric and magnetic fields $\mathbf{E}$ and $\mathbf{B}$ respectively [1], so in dual QCD the dual masses $\widetilde{m}_{\text {mag }}$ and $\widetilde{m}_{\mathrm{el}}$ are associated with the correlation functions of the $\mathbf{H}$ and $\mathbf{D}$ fields respectively. Obviously, in a very complicated dielectric medium such as a quark-gluon plasma, there is no reason to expect any simple relationships between these four different correlation functions.

In perturbation theory $\widetilde{m}_{\text {mag }}$ and the associated dual magnetic plasma frequency $\widetilde{m}_{\text {mag }}$ can be calculated to or$\operatorname{der} g T$, and the associated Landau damping $\widetilde{\gamma}_{\text {mag }}$ in order $g^{2} T$. However, in analogy to ordinary QCD, the dual electric mass $\widetilde{m}_{\mathrm{el}}$ begins in order $g^{2} T$ and cannot be calculated perturbatively.

The physical significance of the dual quantities is that $\tilde{m}_{\text {mag }}$ provides the screening length for the colormagnetic field $\mathbf{H}$, and $\widetilde{\omega}_{\text {mag }}$ gives the plasma oscillation frequency of that field around equilibrium. It is not clear how these quantities would be measured experimentally, but it is also not clear how the analogous electric quantities $m_{\mathrm{el}}$ and $\omega_{\mathrm{el}}$ would be measured either. As we stated above, in a complicated dielectric medium, all four fields, $\mathbf{E}, \mathbf{B}, \mathbf{H}$ and $\mathbf{D}$ are not easily related to each other, and 
all can behave differently in the plasma.

In dual QCD, the one-loop diagrams contribution to the dual gluon inverse propagator (which of course is transverse as in usual QCD) are shown in Fig. 1. The extra graphs involving the dual QCD "Higgs fields" $B_{i}^{a}$ follow from the dual QCD Lagrangian [5]. The quark couplings in the quark loop graph to dual gluons also differ from those in ordinary QCD [4]. However, since in a hot quark-(dual)-gluon plasma, one is above the deconfinement transition temperature, dual gluons (as well as the $B_{i}^{a}$ particles) are massless, so the dual gluon graphs are precisely the same as in ordinary QCD.

The calculation of the graphs in Fig. 1 is straightforward, using the Feynman rules of dual QCD [4]. Because of the form of the quark-dual-gluon coupling, the quark loop in Fig. 1 does not contribute in the limit when the external gluon momentum vanishes (either in the limit first $q_{0} \rightarrow 0$ and then $\mathbf{q} \rightarrow 0$ or vice versa) [6]. The final result turns out to be

$$
\widetilde{m}_{\text {mag }}^{2}=\frac{1}{2} g^{2} T^{2}\left(1+\frac{1}{6}\right)=\frac{7}{12} g^{2} T^{2},
$$

where the second term in the parentheses comes from the "Higgs" loops shown in Fig. 1. Numerically, using the value $g=2.8$ obtained previously [5], we find

$$
\widetilde{m}_{\text {mag }}=2.2 T \text {, }
$$

although we should emphasize that in this energy regime we really do not know what $g$ is.

In the infrared limit, $g$ is related to the usual QCD coupling constant $e$ by $e g=2 \pi$ because dual QCD and ordinary QCD should be the same theory in this limit; at high $T$, this relation no longer holds, and the value of $g$ is unknown. As in the electric case, because of the transversality of $\left(\Pi_{c}\right)_{\mu \nu}$, the magnetic ( $\mathbf{H}$ field) plasma frequency is related to the dual magnetic mass by

[1] D. Gross, R. Pisarski, and L. Yaffe, Rev. Mod. Phys. 53, 43 (1981).

[2] C. P. Burgess and A. L. Marini, Phys. Rev. D 45, R17 (1992).

[3] E. Braaten and R. Pisarski, Phys. Rev. Lett. 64, 1338 (1990); R. Kobes, G. Kunstatter, and A. Rebhan, ibid. 64, 2992 (1990); Nucl. Phys. B355, 1 (1991).

[4] M. Baker, J. Ball, and F. Zachariasen, Phys. Rep. 209, 74

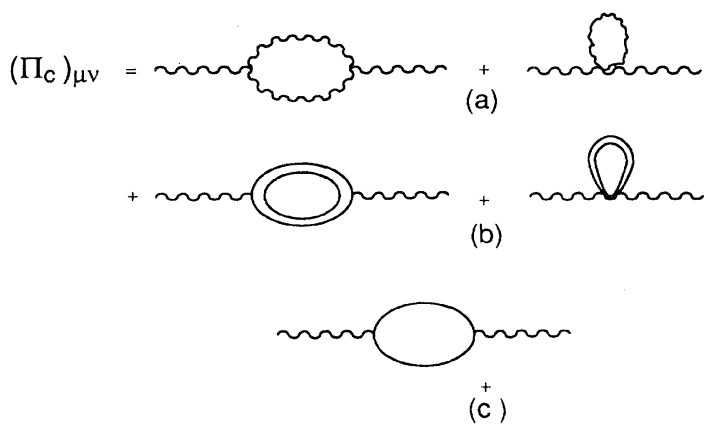

FIG. 1. Diagrams showing the contributions to the dual gluon inverse propagator of (a) dual gluon loops (dual gluons are indicate by wavy lines), (b) "Higgs" loops ("Higgs" particles are shown as double line), and (c) the quark loop (a quark is shown as a single line).

$$
\widetilde{\omega}_{\mathrm{mag}}=\frac{1}{\sqrt{3}} \widetilde{m}_{\mathrm{mag}} .
$$

We have not calculated the value of the dual damping constant $\widetilde{\gamma}_{\text {mag }}$, though it is not hard to do so; we expect the result to be much the same as for $\gamma_{\mathrm{el}}$ with $e$ replaced by $g$.

The work of M.B. was supported in part by the U.S. Department of Energy under Contract No. DOE/ER/40614. The work of J.S.B. was supported in part by the National Science Foundation Grant No. PHY 9008482. The work of F.Z. was supported in part by the U.S. Dept. of Energy under Contract No. DEAC-0381ER40050.
(1991).

[5] M. Baker, J. Ball, and F. Zachariasen, Phys. Rev. D 45, 910 (1992).

[6] The calculation of the $q \rightarrow 0$ quark loop is essentially carried out by M. Baker, J. Ball, and F. Zachariasen, Phys. Rev. D 40, 2732 (1989). The loop given there is multiplied by two powers of $q$ due to the form of the quark-dualgiluon vertex (see Ref. [5]) and so vanishes as $q \rightarrow 0$. 